\title{
HUNDERT JAHRE LANDESHAUPTSTADT BERN
}

\author{
Von Heinrich Gutersohn
}

Mit 2 Abbildungen und 1 Tafel

Die Schweiz feiert 1948 das hundertjährige Bestehen ihrer Verfassung, jenes für unser Land bedeutsamen Verständigungswerkes, das aus dem aristokratischen Staatenbund die demokratische Republik, den Bundesstaat, schuf. Die Stadt Bern aber darf ein Doppeljubiläum begehen, wurde sie doch gleichzeitig zur Bundeshauptstadt erkoren, eine Ehrung, die ihr zweifellos viele Pflichten, aber auch besondere Entwicklungsmöglichkeiten brachte.

Fassen wir kurz die entscheidenden Daten zusammen ${ }^{1}$ : Nachdem am 12. September 1848 die neue Verfassung von Volk und Ständen mit großem Mehr angenommen worden war, trat am 6 . November die erste Bundesversammlung im bisherigen eidgenössischen Vorort Bern zusammen. Sie wählte die Bundesräte, und am 28. November bestimmte sie Bern zur Bundeshauptstadt. Bern erklärte am 18. Dezember 1848 Annahme seiner neuen Stellung. Der Wahl war ein harter Konkurrenzkampf namentlich zwischen Bern und Zürich vorausgegangen. Wir Zürcher blicken heute auf unsere bundesstädtische Jubilarin mit der vollen Überzeugung, daß der damalige Entscheid richtig war. Er wies jeder der beiden Städte ihre besondere Hauptaufgabe zu: Bern die Bundesverwaltung, Zürich Handel und Industrie. Mit diesen, ihren Hauptfunktionen aber war jedem der beiden Zentren die Möglichkeit einer eigenständigen Entwicklung gegeben. Volles Entfalten der individuellen Eigenart und damit Verhindern der Nivellierung gibt der einzelnen Stadt erst die Möglichkeit, wertvolles Glied des Ganzen zu sein. Der Bundesstaat Schweiz mag ruhig aus heterogenen Landschaften gefügt sein; wenn sie alle sich harmonisch ergänzen, verbürgt ja gerade die Vielheit erst die größte innere Stärke.

Bern war bis vor hundert Jahren die geschlossene, 40-60 m über der eingeschnittenen Aare stehende Halbinselstadt geblieben, etwa so, wie sie auf dem beigelegten phantasievollen Farbenkärtchen dargestellt ist ${ }^{2}$. Von der östlichen Enge des Gleitufers aus divergieren drei Längsgassen bis zum Kornhausplatz mit seinem Zeitglockenturm, das heißt bis zum ehedem landseitigen, bis 1255 vorhandenen Querabschluß. Ihnen schließen sich vier Längsgassen an bis zum Bären- und Waisenhausplatz, wo die erste Stadterweiterung um 1346 durch einen Graben endete. Und dann erweitert sich das Füllhorn der alten Gassen auf sieben, bis zu dem durch Graben und Schanzen gesicherten Querriegel der zweiten Stadterweiterung von 1623, wo heute Bahnhof und Burgerspital stehen. Im Aufrißbild der Stadt dominierten das imposante Münster und weitere Kirchtürme, die gemauerte Münsterterrasse, die geschlossenen, durch Bauvorschriften geschützten Fronten der zusammengebauten Häuser mit den selbstbewußten Fassaden der alten Patrizierbauten und den durchgehenden gemütlichen Laubengängen beidseits der Hauptgassen, alles mit einheitlichem Material, dem bernischen Sandstein, erbaut. Isoliert von der geschlossenen Stadt blieben nur die gewerblichen Bauten in der Matte und relativ wenige weitere Häuser beidseits der Aare (Bild 1). Daß uns dieses alte Bern trotz der mächtigen Stadtentwicklung im großen und ganzen in seiner Eigenart erhalten blieb, ist überaus erfreulich.

Die neue Funktion als Landeshauptstadt brachte Bern 1858 das erste Bundeshaus, den heutigen Westflügel, 1892 den Ostbau und schließlich 1902 den Kuppelbau des Parlamentsgebäudes als verbindendes Zentrum. Sie brachte aber auch Beamte, die

1 Historisch-Biographisches Lexikon der Schweiz. Neuenburg. Stichworte "Bern" und "Schweiz".

2 Vgl. Wyss, FrITz: Bern, eine kleine Stadtgeographie. Bern 1943. 88 Seiten, mit Literaturverzeichnis. 


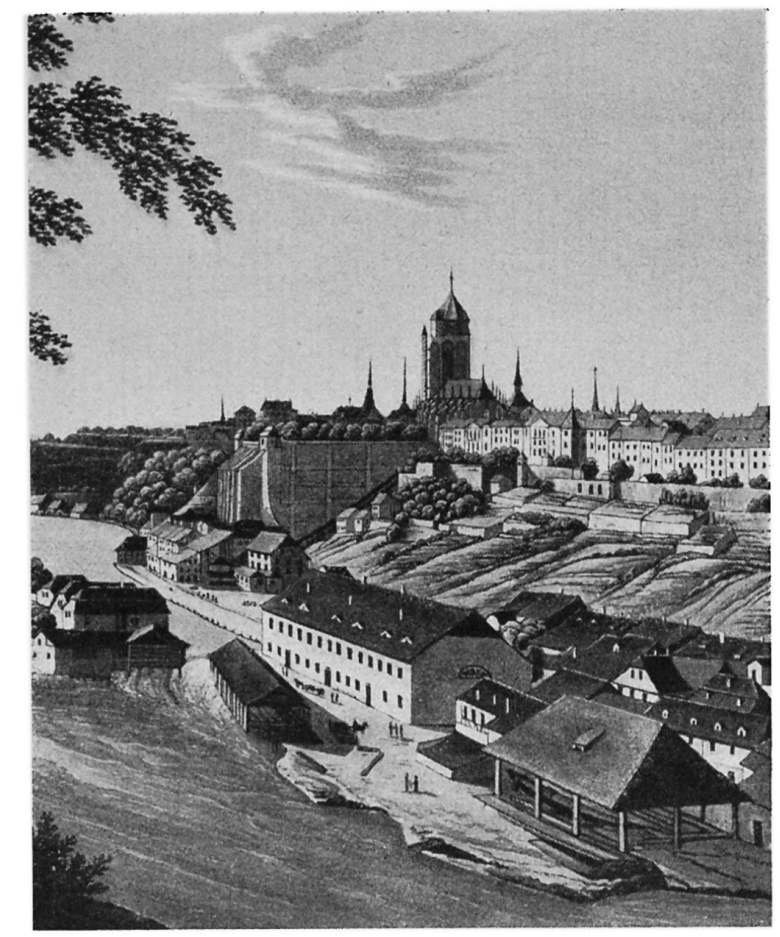

Bild 1. Ansicht von Bern vom Aargauer Stalden aus, um 1850. Über die geschlossene Front der Stadt ragt neben den $40 \mathrm{~m}$ hohen Mauern der Münsterterrassc das Münster empor. Im Vordergrund, am Ufer der Aare, Wasserwerke, gewerbliche Anlagen und Wohnhäuser der Matte.

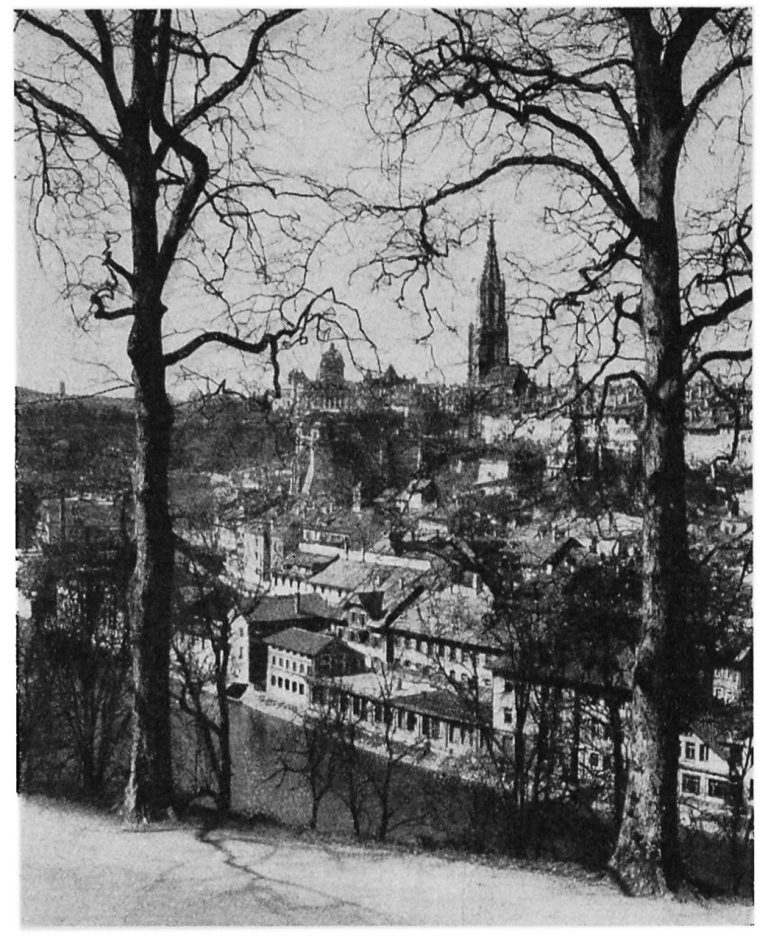

Bild 2. Ansicht von Bern vom gleichen Standort aus wic Bild 1, um 1940. Das Quartier Matte ist teilweise umgebaut, der Hang der Halbinsel mit weiteren Häusern besetzt, das Münster hat den vollständig ausgebauten 'Turm erhalten. Links ist die Kuppel des Bundeshauses. 
Wohnungen benötigten. Und überdies setzte ja gleichzeitig jene ungestüme Entwicklung von Industrie und Verkehr ein, die auch anderwärts rasche Vergrößerungen des Stadtgrundrisses verlangte. Indem man zunächst auf der Landseite jenseits der schon 1834-1845 größtenteils geschleiften Schanzen Neubauten errichtete, entstanden Anfänge der Stadtteile Brückfeld, Länggasse, Stadtbach und Villette, denen sich später weitere, äußere angliederten. Offenen Raum aber boten die Schotterfelder ennet der Aareschleife, für deren Bebauung die Eröffnung neuer Brücken zu eigentlichen Initialzündungen wurden.

Nicht daß vorher überhaupt keine Übergänge bestanden hätten; schon seit 1256 setzte die Untertorbrücke vom östlichen Stadtende aus über den Fluß, und drüben erklomm die Landstraße in langer Rampe wieder die Höhe, bevor sie frei über das Feld weiterführte. 1834 wurde die von der nördlichen Stadtmitte ans Gegenufer leitende Fähre durch eine weitere Brücke ersetzt, und schon hub auch langsam die Entwicklung des Gegenquartiers Altenberg an. Wirklich gelöst von der Engnis wurde die Altstadt aber erst durch die neuen Hochbrücken, die Altstadtboden und äußere Felder über den Aarecañon direkt verbanden. Als erste konnte 1844 die Nydeggbrücke, hoch über der Untertorbrü̈ke, dem Verkehr übergeben werden, ohne daß allerdings darauf eine rasche Besiedlung des Nachbarquartiers Schoßhalden gefolgt wäre. Anders dagegen, als 1858 die Rote Brücke in die Lorraine hinüberleitete, die in erster Linie der neuen Eisenbahn, aber mit einer besonderen Fahrbahn auch Fuhrwerken und Fußgängern diente. Jetzt entstand rasch das Quartier Lorraine, anschließend Breitenrain und Spitalacker. Bald genügte die Rote Brücke nicht mehr; es folgte 1898 die Kornhaus- und 1930 eine neue Lorrainebrücke. Inzwischen war auch die Überwindung des Südrandes der Stadt gelungen. Eine englische Gesellschaft öffnete 1883 die auf eigene Kosten erstellte Kirchenfeldbrücke dem Verkehr. Drüben im Kirchenfeld stellte sie Interessenten im Rahmen eines festgelegten Quartierplanes Bauland zur Verfügung, wodurch ein villenartiges Quartier entstand. Wenn auch später die Gestaltung nicht in allen Teilen der Planung entsprach, so wuchs hier ein Teil des neuen Berns doch nach Richtlinien, die heute, wo mit Recht der Ortsplanung immer größeres Gewicht beigemessen wird, durchaus modern anmuten.

Bern, vorher in den allzu engen Schlauch der Halbinsel hineingezwängt, entfaltete sich so dank seiner Brücken auf allen Seiten, und sein Grundriß nähert sich zusehends dem Kreis. Wohnten 1850 in den rund 1200 Wohnhäusern der Altstadt erst 27558 Einwohner, so waren es $1941 \mathrm{im}$ größeren Bern deren 130331 in 10868 bewohnten Häusern. Dabei ist nur eine einzige Eingemeindung, nämlich 1919 jene von Bümpliz, erfolgt. Im ungestümen Drang nach außen blieben allerdings einige ungünstiger gelegene Quartiere zurück, so die auf der flachen Niederung des Gleitufers mit Wohnund gewerblichen Bauten besetzte Matte. Die neueste Zeit greift aber auch hier ein, holt Versäumtes nach und ersetzt baulich unbefriedigende Quartiere durch neue (Bild 2).

Wenn auch Bern kein eigentliches Industriequartier besitzt, so ist die Industrie doch in den Außenquartieren gut vertreten. Von den 67198 Berufstätigen (1941) fanden $37 \%$ ihr Auskommen in Industrie und Handwerk, 30\% in Handel, Gastgewerbe und Verkehr, knapp $1 \%$ in der Landwirtschaft. Von den restlichen $32 \%$ war zweifellos der größte Teil in der Verwaltung beschäftigt. Dieser wichtigen Funktion dienen nicht nur zahlreiche Gebäude von Gemeinde und Kanton, sondern natürlich vor allem die vielen Verwaltungsbauten der Eidgenossenschaft, vom Bundeshaus über die übrigen Amtsgebäude bis zu den kriegsbedingten Notbaracken im Marzili. Beträchtliche Räume nehmen auch die als Folge der zentralen Bedeutung Berns weiteren notwendigen Dienste ein: die Kaufläden vor allem in der Altstadt, Gebäude von Hochschule und Zentralverwaltung der Schweizerischen Bundesbahnen auf der Großen Schanze, Spitäler im Friedbühlquartier, militärische Bauten und Plätze im Beundenfeld u. a. Der an den Nordwestrand der Stadt grenzende prächtige Bremgartner Wald darf füglich als 


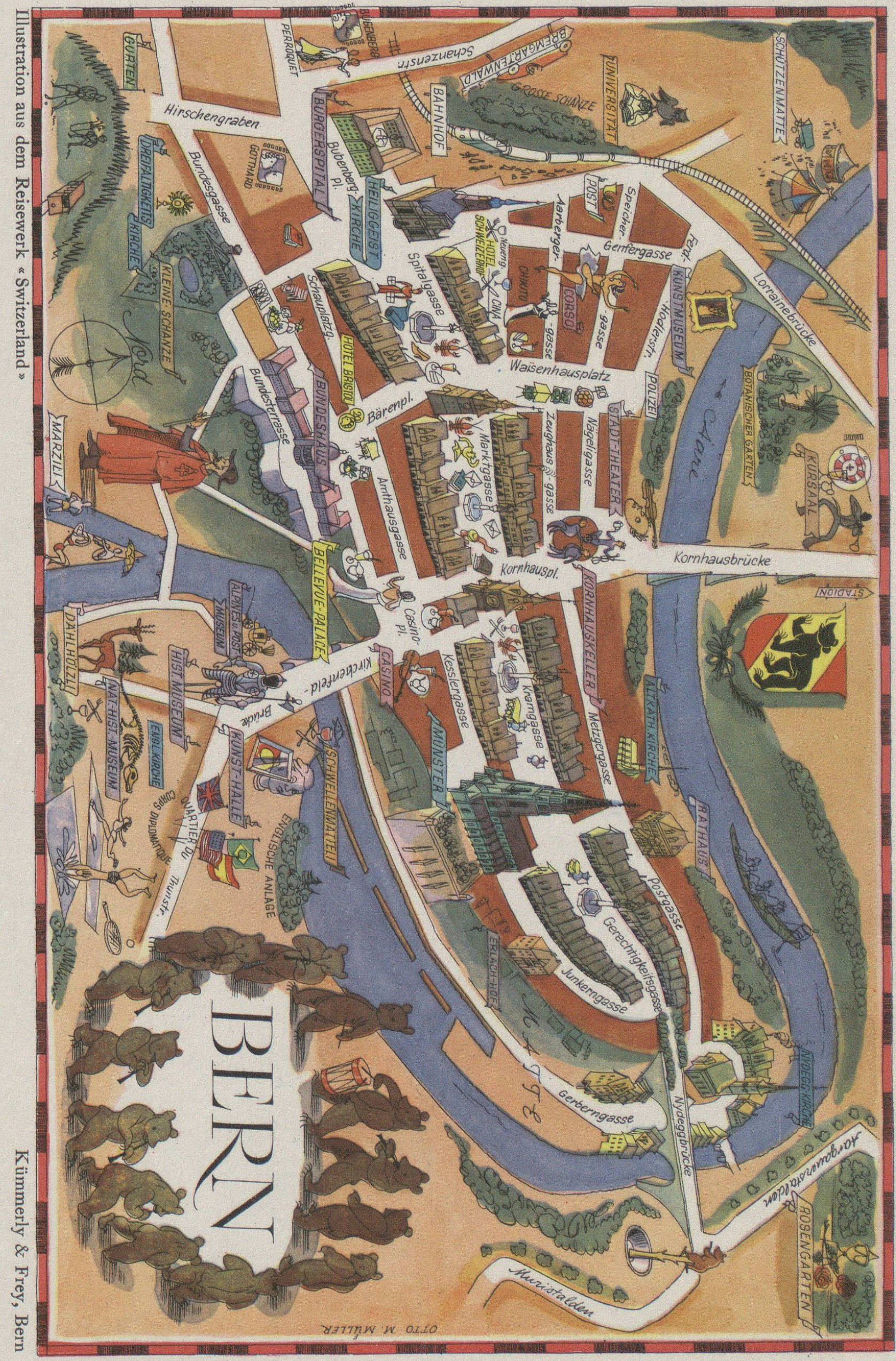


modernes Erholungsgebiet gelten, das durch weitere, wie Gurten und Könizwald, ergänzt wird. Überall aber breiten sich ausgedehnte Wohnquartiere aus, unter denen die in jüngster Zeit mit imponierendem städtischem Unternehmungsgeist geschaffenen Wohnkolonien das Streben nach gesunder städtebaulicher Entwicklung belegen.

Wir Geographen sind uns in besonderem Maße der Tatsache eingedenk, daß das Wohlergehen der Menschen weitgehend davon abhängt, ob sie in einer optimal organisierten, harmonischen Landschaft leben können. Wir werden deshalb auch in Zukunft aufmerksam auf Bern schauen. In Bern wurde vor achtzig Jahren die damals umfassendste praktische Landschaftsgestaltung, die Juragewässerkorrektion, durchdacht und gelenkt, und viele weitere Entscheide fielen seither in seinen Mauern, die die Weiterentwicklung der schweizerischen Kulturlandschaft ebenfalls grundlegend beeinflußten. Wir hegen die Hoffnung, daß sich die verantwortlichen Männer in unserer lieben Bundesstadt auch künftig dieser Bedeutung ihrer Entschlüsse bewußt seien.

\section{BERNE, VILLE FÉDÉRALE DEPUIS CENT ANS}

L'accroissement de Berne depuis cent ans dépend sans doute de sa fonction comme capitale de la Suisse, mais aussi du développement prodigieux de l'industrie, du commerce et de sa circulation. En 1848 encore, la ville était enserrée dans l'étroit boucle de l'Aar. Mais depuis lors elle s'est étendue, surtout grâce aux quatre nouveaux ponts reliant les nappes de graviers supérieures; son plan s'approche de plus en plus de la forme circulaire.

\section{CENTENARIO DELLA CAPITALE FEDERALE BERNA}

L'accrescimento di Berna nel corso degli ultimi cento anni è dovuto in gran parte alla sua funzione come capitale della Confederazione, ma anche allo sviluppo tempestivo della sua industria, del commercio e del traffico. Mentre nel 1848 la superfice della città si limitava di coprire soltanto la stretta penisola dell'Aare, oggi grazie ai quattro ponti questa si è dilatata oltre le ghiaie delle pianure esterne e si avvicina ad una forma circolare.

\section{MEIN STANDPUNKT IN DER ORTSNAMENFRAGE}

\section{Von EDUARD IMHOF}

J. Hubschmid setzte sich in der "Geographica Helverica», II, 1947, Heft 4, für eine mundartliche Schreibung der deutschschweizerischen Orts- oder Lokalnamen ein. Er wies hin auf meine Abhandlung über «Die Ortsnamen in den amtlichen Plänen und Karten» (Schweizerische Zeitschrift für Vermessungswesen und Kulturtechnik, 1945, Heft 5-9), und er hob einiges Gemeinsames in unsern Auffassungen hervor. Dies täuscht den Leser über die nur leicht angedeutete Tatsache grundsätzlicher Verschiedenheit unserer Vorschläge hinweg. Mein Standpunkt in der Ortsnamenfrage sei daher im folgenden kurz dargelegt.

Eine den lokalen Mundarten möglichst angenäherte Schreibung würde in extremen Fällen zu Namen folgender Art führen (die entsprechenden, bisher üblichen Formen sind in Klammern beigefügt): Chilefäldbrugg (Kirchenfeldbrücke), Chleini Ämme (Kleine Emme), Underalpriiß (Unteralpreuß), Chlöntel (Klöntal), Hooli Gaß (Hohle Gasse), Tiifelsbrigg (Teufelsbrücke), Chrüzbärg (Kreuzberge), Höch Chaschte (Hoher Kasten), Miliwäg (Mühleweg), Glai Baasel (Klein-Basel), Ußersiil (Außersihl), Fiischteraarfire (Finsteraarfirn), Schregghore (Schreckhorn), Niderbue (Niederbauen).

Solche sprachlichen Roßkuren brächten die dringend notwendige Nomenklaturrevision in Mißkredit. Es trifft nicht zu, daß solche Formen die Verständigung erleichtern oder daß sie der Deutschschweizer in seinen Plänen und Karten begrüßen würde. Sein Sinn für das Praktische und seine Angewöhnung an gewisse allägliche Schreibformen sträuben sich dagegen. Auch ich befürworte durchaus eine sprachlich bereinigte Anpassung an die heutigen lokalen Sprechformen, bin aber überzeugt, daß die Sache ohne gewisse Konzessionen zu heillosen Verwirrungen und Erschwerungen führen 